\title{
INEQUALITIES SATISFIED BY A CERTAIN DEFINITE INTEGRAL
}

BY G. H. HARDY AND NORMAN LEVINSON*

1. Introduction. In this note we solve the following problem. Suppose that

$$
\begin{aligned}
0 & \leqq a_{1}<a_{2}<\cdots<a_{2 n+1} \leqq 1, \\
f(x) & =\frac{\left(x-a_{2}\right)\left(x-a_{4}\right) \cdots\left(x-a_{2 n}\right)}{\left(x-a_{1}\right)\left(x-a_{3}\right) \cdots\left(x-a_{2 n+1}\right)}, \\
J(t) & =\int_{0}^{1}|f(x)|^{t} d x, \quad 0<t<1 .
\end{aligned}
$$

Then what are the best inequalities satisfied by $J(t)$ ?

We prove the following theorem:

Theorem A. If $f(x)$ satisfies (1) then

$$
\frac{\Gamma\left(\frac{1}{2}+\frac{1}{2} t\right) \Gamma\left(1-\frac{1}{2} t\right)}{(1-t) \pi^{1 / 2}} \leqq J(t) \leqq \frac{2^{t}}{1-t},
$$

with inequality except when

$$
\begin{aligned}
f(x) & =\frac{1}{x-\frac{1}{2}}, & J(t) & =\frac{2^{t}}{1-t} \\
f(x) & =\frac{x-\frac{1}{2}}{x(x-1)}, & J(t) & =\frac{\Gamma\left(\frac{1}{2}+\frac{1}{2} t\right) \Gamma\left(1-\frac{1}{2} t\right)}{(1-t) \pi^{1 / 2}} .
\end{aligned}
$$

The integral $J(t)$ occurred in a recent paper by Levinson. $\dagger$ Levinson proved that

and indeed that

$$
J(t)<\frac{5}{1-t}
$$

$$
\int_{0}^{1}|f(x+i y)|^{t}<\frac{5}{1-t}
$$

* National Research Fellow.

$\dagger$ Levinson, On non-harmonic Fourier series, Annals of Mathematics, (2), vol. 37 (1936), p. 922. 
for any real $y$, and stated without proof a more precise, though still not the best possible, inequality. Here we confine ourselves to the case $y=0$, but our results are the best of their kind. We prove them by two methods, one "real" and one "complex".

2. A Theorem of Boole. Lemma 1. If $f(x)$ satisfies (1), then

$$
\int_{-\infty}^{\infty} F\{f(x)\} d x=\int_{-\infty}^{\infty} F(y) \frac{d y}{y^{2}}
$$

whenever (i) $F(y)$ is defined for all values of $y$, and (ii) either integral exists as a Lebesgue integral.

Lemma 1 is essentially the same as a theorem of Boole.*

There are two other definitions of $f(x)$ equivalent to that of $\S 1$. In the first place, as we can verify at once by resolving $f(x)$ into partial fractions,

$$
f(x)=\sum_{\nu=0}^{n} \frac{\alpha_{\nu}}{x-a_{2 \nu+1}}
$$

where

$$
\alpha_{\nu}>0, \quad \sum \alpha_{\nu}=1
$$

This is the form which we shall generally use here. Secondly

$$
g(x)=\frac{1}{f(x)}=x-\sum_{\nu=0}^{n} \frac{\beta_{\nu}}{x-a_{2 \nu}},
$$

where $\beta_{\nu}>0$. If we write $1 / y$ for $y$ and $G(y)$ for $F(1 / y)$, then (2) becomes

$$
\int_{-\infty}^{\infty} G\{g(x)\} d x=\int_{-\infty}^{\infty} G(y) d y,
$$

which is Boole's formula.

To prove Lemma 1 we observe that, after (3) and (4), the graph of $f(x)$ consists of $n+2$ descending pieces corresponding to the intervals $\left(-\infty, a_{1}\right),\left(a_{1}, a_{3}\right), \cdots,\left(a_{2 n+1}, \infty\right)$, the corresponding intervals of variation of $f(x)$ being $(0,-\infty)$,

* G. Boole, On the comparison of transcendents, with certain applications to the theory of definite integrals, Philosophical Transactions of the Royal Society, vol. 147 (1857), pp. 745-803. See in particular p. 780. Boole's very interesting memoir has been forgotten, and his results have been rediscovered, wholly or in part, by a number of later mathematicians. 
$(\infty,-\infty), \cdots,(\infty, 0)$; and that, when $x$ moves from $-\infty$ to $\infty, y$ moves, in all, $n+1$ times over the same range. The line $f(x)=y$ cuts the graph of $f(x)$ in $n+1$ points $x_{1}, x_{2}, \cdots, x_{n+1}$; and

$$
\int_{-\infty}^{\infty} F(y) d x=\int_{-\infty}^{\infty} F(y) P(y) \frac{d y}{y^{2}},
$$

where

$$
P(y)=-y^{2} \sum_{\nu}\left(\frac{d x}{d y}\right)_{x=x_{\nu}}
$$

We have to prove that*

$$
P(y)=1 \text {. }
$$

It is plain that, if $f(x)=y$, then

$$
y \prod_{\nu}\left(x-a_{2 \nu+1}\right)-\sum_{\nu} \alpha_{\nu} \prod_{\mu \neq \nu}\left(x-a_{2 \mu+1}\right)=y \prod_{\nu}\left(x-x_{\nu}\right) .
$$

Hence, first, equating the coefficients of $x^{n-1}$ and using (4), we have

$$
\sum x_{\nu}-\sum a_{2 \nu+1}=\frac{1}{y} .
$$

Next, (6) is an identity in $y$ when $x_{\nu}(y)$ is substituted for $x_{\nu}$. Hence, differentiating this, we obtain

$$
\sum \frac{d x_{\nu}}{d y}=-\frac{1}{y^{2}} .
$$

It follows that $P(y)=1$.

3. The Underlying Identity. In what follows it is convenient to symmetrize our analysis about the origin, which we can do by writing $x-\frac{1}{2}$ for $x$. We have then

(7) $J(t)=\int_{-1 / 2}^{1 / 2}|f(x)|^{t} d x, f(x)=\sum \frac{\alpha_{\nu}}{x-a_{2 \nu-1}}, \alpha_{\nu}>0, \sum \alpha_{\nu}=1$, and

$$
-\frac{1}{2} \leqq a_{1}<a_{2}<\cdots<a_{2 n+1} \leqq \frac{1}{2} .
$$

\footnotetext{
* We are indebted to Professor Bohnenblust for a simplification of the proof.
} 
Lemma 2. If $f(x)$ satisfies (7) and (8), then

(9) $J(t)=\frac{2^{t}}{1-t}-\int_{1 / 2}^{\infty}\left\{|f(x)|^{t}+|f(-x)|^{t}-\frac{2}{x^{t}}\right\} d x$.

Suppose that $\epsilon$ is small and positive and that $\xi$ and $\eta$ are the largest and smallest roots of $f(x)=\epsilon$ and $f(x)=-\epsilon$ respectively. Then $\xi>\frac{1}{2}$ and $\eta<-\frac{1}{2}$. Also

$$
\frac{1}{\xi+\frac{1}{2}} \leqq \sum \frac{\alpha_{\nu}}{\xi-a_{2 v+1}}=\epsilon \leqq \frac{1}{\xi-\frac{1}{2}},
$$

and so

$$
\begin{gathered}
\frac{1}{\epsilon}-\frac{1}{2} \leqq \xi \leqq \frac{1}{\epsilon}+\frac{1}{2}, \\
\xi=\frac{1}{\epsilon}+O(1),
\end{gathered}
$$

where the $O$ refers to the limit process $\epsilon \rightarrow 0$. Similarly

$$
\eta=-\frac{1}{\epsilon}+O(1) \text {. }
$$

Define $f_{\epsilon}$ by the relations

$$
f_{\epsilon}=f, \quad(|f| \geqq \epsilon) ; \quad f_{\epsilon}=0, \quad(|f|<\epsilon) .
$$

Then, by Lemma 1 ,

$$
\int_{-\infty}^{\infty}\left|f_{\epsilon}\right| t d x=2 \int_{\epsilon}^{\infty}|y|^{t-2} d y=\frac{2 \epsilon^{t-1}}{1-t} .
$$

Hence

$$
\begin{aligned}
J(t) & =\int_{-1 / 2}^{1 / 2}|f|^{t} d x=\lim _{\epsilon \rightarrow 0} \int_{-1 / 2}^{1 / 2}\left|f_{\epsilon}\right|^{t} d x \\
& =\lim _{\epsilon \rightarrow 0}\left\{\frac{2 \epsilon^{t-1}}{1-t}-\left(\int_{1 / 2}^{\xi}|f|^{t} d x+\int_{\eta}^{-1 / 2}|f|^{t} d x\right)\right\} .
\end{aligned}
$$

Now

$$
f(x)=x^{-1}+O\left(x^{-2}\right), \quad|f(x)|^{t}=|x|^{-t}+O\left(|x|^{-t-1}\right)
$$


for large $x$. Hence, by (10),

$$
\begin{aligned}
\int_{1 / \epsilon}^{\xi}|f|^{t} d x & =\frac{1}{1-t}\left\{\left(\frac{1}{\epsilon}+O(1)\right)^{1-t}-\left(\frac{1}{\epsilon}\right)^{1-t}\right\}+O\left(\epsilon^{t}\right) \\
& =O\left(\epsilon^{t}\right)
\end{aligned}
$$

and we may replace $\xi$ by $1 / \epsilon$ in (12). Similarly we may replace $\eta$ by $-1 / \epsilon$. Hence

$$
\begin{aligned}
J(t)= & \lim _{\epsilon \rightarrow 0}\left\{\frac{2 \epsilon^{t-1}}{1-t}-\int_{1 / 2}^{1 / \epsilon}\left\{|f(x)|^{t}+|f(-x)|^{t}\right\} d x\right\} \\
=\lim _{\epsilon \rightarrow 0}\left\{\frac{2 \epsilon^{t-1}}{1-t}-\right. & 2 \int_{1 / 2}^{1 / \epsilon} \frac{d x}{x^{t}} \\
& \left.-\int_{1 / 2}^{1 / \epsilon}\left\{|f(x)|^{t}+|f(-x)|^{t}-\frac{2}{x^{t}}\right\} d x\right\},
\end{aligned}
$$

which is (9).

4. A Lemma. Lemma 3. If $|x|>\frac{1}{2}$ then

$$
\phi(x)=|f(x)|^{t}+|f(-x)|^{t}
$$

is (for every $x$ ) least and greatest when $f(x)$ is $1 / x$ and $x /\left(x^{2}-\frac{1}{4}\right)$ respectively.

We may suppose $x>\frac{1}{2}$. We consider the pole $A$ of $f(x)$ nearest to an end of $\left(-\frac{1}{2}, \frac{1}{2}\right)$. If we suppose, for example, that $A>0$, then $A=a_{2 n+1}$. If

$$
\xi=\frac{1}{x-a}, \quad \xi^{\prime}=\frac{1}{x+a}, \quad \Xi=\frac{1}{x-A}, \quad \Xi^{\prime}=\frac{1}{x+A},
$$

then all these numbers are positive and

$$
\frac{\Xi}{\Xi^{\prime}}>\frac{\xi}{\xi^{\prime}} \geqq 1
$$

for any pole $a$ other than $A$. If

$$
\begin{aligned}
\psi(A) & =\phi(x)=|f(x)|^{t}+|f(-x)|^{t} \\
& =\left(\sum \frac{\alpha}{x-a}\right)^{t}+\left(\sum \frac{\alpha}{x+a}\right)^{t},
\end{aligned}
$$


then

$$
\frac{1}{t} \frac{d \psi(A)}{d A}=|f(x)|^{t-1} \frac{\mathrm{A}}{(x-A)^{2}}-|f(-x)|^{t-1} \frac{\mathrm{A}}{(x+A)^{2}},
$$

where $\mathrm{A}$ is the $\alpha$ corresponding to $A$. This will be positive if

$$
\left(\frac{\Xi}{\Xi^{\prime}}\right)^{2}>\left(\frac{\sum \alpha \xi}{\sum \alpha \xi^{\prime}}\right)^{1-t}
$$

and this is true on account of (13).

Hence we decrease $\phi(x)$ by moving $A$ to the left, to the next pole, or to the origin if there is no other positive pole. Similarly, if $A$ were negative, we should decrease $\phi(x)$ by moving $A$ to the right. It follows by repetition of the argument that $\phi(x)$ is least when all the $a$ 's coincide at the origin, and $f(x)=1 / x$.

Similarly $\phi(x)$ is greatest when all the $a$ 's are at one of the ends of $\left(-\frac{1}{2}, \frac{1}{2}\right)$. In this case

$$
f(x)=\frac{\alpha}{x-\frac{1}{2}}+\frac{1-\alpha}{x+\frac{1}{2}}=\frac{x-\beta}{x^{2}-\frac{1}{4}}
$$

where $\beta=\alpha-\frac{1}{2}, 0 \leqq \alpha \leqq 1,|\beta| \leqq \frac{1}{2}$. Finally

$$
|x-\beta|^{t}+|x+\beta|^{t}<2|x|^{t}
$$

if $|x|>\frac{1}{2}, \beta \neq 0$, so that the true maximum of $\phi(x)$ occurs when

$$
f(x)=\frac{x}{x^{2}-\frac{1}{4}}
$$

5. Proof of the Inequalities. We can now prove the theorem. We take the interval as $\left(-\frac{1}{2}, \frac{1}{2}\right)$, so that the two critical functions are

$$
f_{1}(x)=\frac{1}{x}, \quad f_{2}(x)=\frac{x}{x^{2}-\frac{1}{4}}
$$

It follows from (9) and Lemma 3 that

$$
J(t) \leqq \frac{2^{t}}{1-t},
$$

with inequality unless $f=f_{1}$. Also 


$$
\begin{aligned}
& \int_{-1 / 2}^{1 / 2}|f| t d x-\int_{-1 / 2}^{1 / 2}\left|f_{2}\right|^{t} d x \\
& \quad=\int_{1 / 2}^{\infty}\left(\left|f_{2}(x)\right|^{t}+\left|f_{2}(-x)\right|^{t}-|f(x)|^{t}-|f(-x)|^{t}\right) d x
\end{aligned}
$$

by (9), and the last integral is positive, by Lemma 3, unless $f=f_{2}$. Finally

$$
\int_{-1 / 2}^{1 / 2}\left|f_{2}\right|^{t} d x=\int_{-1 / 2}^{1 / 2}\left|\frac{x}{x^{2}-\frac{1}{4}}\right|^{t} d x=\frac{\Gamma\left(\frac{1}{2}+\frac{1}{2} t\right) \Gamma\left(1-\frac{1}{2} t\right)}{(1-t) \pi^{1 / 2}},
$$

by an elementary calculation.

6. Alternative Proof of the Underlying Identity. There is another proof of (9) by complex integration. We integrate

$$
\int\left\{(f(x))^{t}-\frac{1}{x^{t}}\right\} d x
$$

around a contour $C$ composed of (i) small semicircles of radius $\rho$, above the real axis, around the singularities $a_{k}$ and 0 , (ii) a large semicircle of radius $R$, above the real axis, around 0 , and (iii) the parts of the real axis between these semicircles. We suppose

$$
(f(x))^{t}>0, \quad x^{t}>0
$$

for large positive $x$, and make $\rho \rightarrow 0$ and $R \rightarrow \infty$ in the usual manner. Then $(f(x))^{t}$ is positive along

$$
\left(a_{1}, a_{2}\right)\left(a_{3}, a_{4}\right), \cdots,\left(a_{2 n+1}, \infty\right)
$$

and has the argument of $e^{-t \pi i}$ on the rest of the axis, while $x^{t}$ is positive for $x>0$ and has the argument of $e^{-t \pi i}$ for $x<0$. We thus obtain

$$
I_{1}(t)+e^{-t \pi i} I_{2}(t)=0,
$$

where

$$
\begin{aligned}
I_{1}(t)= & \left(\int_{a_{1}}^{a_{2}}+\int_{a_{3}}^{a_{4}}+\cdots+\int_{a_{2 n-1}}^{a_{2 n}}+\int_{a_{2 n+1}}^{1 / 2}\right)|f(x)|^{t} d x \\
& -\int_{0}^{1 / 2} \frac{d x}{|x|^{t}}+\int_{1 / 2}^{\infty}\left(|f(x)|^{t}-\frac{1}{|x|^{t}}\right) d x
\end{aligned}
$$




$$
\begin{aligned}
I_{2}(t)= & \left(\int_{-1 / 2}^{a_{1}}+\int_{a_{2}}^{a_{3}}+\cdots+\int_{a_{2 n}}^{a_{2 n+1}}\right)|f(x)|^{t} d x \\
& -\int_{-1 / 2}^{0} \frac{d x}{|x|^{t}}+\int_{-\infty}^{-1 / 2}\left(|f(x)|^{t}-\frac{1}{|x|^{t}}\right) d x .
\end{aligned}
$$

If we equate imaginary parts in (14) we obtain

$$
\begin{aligned}
\left(\int_{-1 / 2}^{a_{1}}+\int_{a_{2}}^{a_{3}}+\cdots+\right. & \left.\int_{a_{2 n}}^{a_{2 n+1}}\right)|f(x)|^{t} d x \\
& =\frac{2^{t-1}}{1-t}-\int_{1 / 2}^{\infty}\left(|f(-x)|^{t}-\frac{1}{|x|^{t}}\right) d x
\end{aligned}
$$

and if we multiply by $e^{t \pi i}$, and equate imaginary parts, we obtain

$$
\begin{aligned}
\left(\int_{a_{1}}^{a_{2}}+\int_{a_{3}}^{a_{4}}+\cdots+\int_{a_{2 n+1}}^{1 / 2}\right)|f(x)|^{t} d x \\
=\frac{2^{t-1}}{1-t}-\int_{1 / 2}^{\infty}\left(|f(x)|^{t}-\frac{1}{|x|^{t}}\right) d x .
\end{aligned}
$$

Finally (9) follows by addition.

Institute for Advanced Study and

Princeton University 